\title{
La base de données Baobab : un outil pour modéliser la production et la santé des petits ruminants dans les systèmes d'élevage traditionnels au Sénégal
}

\author{
R. Lancelot ${ }^{1 *}$ B. Faye ${ }^{2}$ X. Juanès ${ }^{2}$ \\ M. Ndiaye ${ }^{1}$ L. Pérochon ${ }^{3}$ E. Tillard ${ }^{4}$
}

Mots-clés

O vin - Caprin - Banque de données Système d'information - Modèle Productivité - Santé animale - Sénégal.

\begin{abstract}
Résumé
La base de données relationnelles Baobab a été construite pour faciliter les travaux de synthèse et de modélisation sur la production et la santé des petits ruminants en élevage sédentaire traditionnel au Sénégal. Elle regroupe les données recueillies dans quatre sites représentatifs des principales zones agroécologiques d'élevage ovin et caprin, pendant 15 ans de suivis d'élevages utilisant le système d'information Panurge. Les données individuelles sont relatives à la démographie, à la croissance pondérale et à la santé. Des données sur les pratiques d'élevage et les prophylaxies collectives ont été recueillies à l'échelle du troupeau. Le modèle conceptuel des données (Mcd) a été élaboré selon la méthode entité-relation Merise. La structure de la base de données a été déduite du Mcd. Les données ont été importées depuis Panurge dans la nouvelle base de données à l'aide de programmes comportant des procédures de correction et de contrôles. Baobab comporte 28 tables et occupe un espace disque d'environ 50 méga-octets pour 79000 animaux recensés. Une nouvelle application a été dérivée du Mcd. Elle permettra de saisir et d'exploiter plus facilement les données provenant de suivis zootechniques individuels, en systèmes sédentaires ou transhumants. Les perspectives d'utilisation de Baobab sont nombreuses et s'orientent selon deux axes principaux : i) la production de référentiels et de synthèses utilisables par les organisations de producteurs et les projets de développement, et permettant les comparaisons entre pays et zones agroécologiques; ii) la modélisation de la production animale, à l'échelle individuelle (génétique, épidémiologie) ou du troupeau (dynamique de population, aide à la décision).
\end{abstract}

\section{INTRODUCTION}

Jusqu'au début des années 1980, les connaissances sur la productivité des petits ruminants dans les conditions d'élevage de l'Afrique intertropicale étaient peu précises. A cette époque, peu

1. Isra-Lnerv, BP 2057, Dakar-Hann, Sénégal

2. Cirad-emvt, Campus international de Baillarguet, BP 5035, 34032 Montpellier Cedex 1, France

3. Inra, Centre de Theix, 63122 Saint-Genès Champanelle, France

4. Cirad-emvt, station Irat, 97410 Saint-Pierre, Réunion

* Auteur pour la correspondance

Tél. : 2218324902 ; fax : 2218211879

E-mail : renaud.lancelot@cirad.fr de données avaient été publiées sur la productivité des ovins et des caprins en milieu villageois $(1,16,34,38,39,40,43,54,55$, 56, 57, 58, 59). Par ailleurs, certaines de ces études portaient sur des échantillons limités et n'abordaient pas tous les aspects de la productivité. Au Sénégal, les rares données publiées avaient été obtenues en station $(6,7)$ et ne concernaient que les ovins Djallonké.

Les sécheresses et les épidémies de peste bovine des années 1970 et 1980 ont entraîné un regain d'intérêt pour les petits ruminants. Par ailleurs, cette époque a vu l'émergence de l'approche systémique, concrétisée au Sénégal par le projet des Unités expérimentales du Siné-Saloum qui intégrait aussi les aspects zootechniques $(22,30)$. En Côte d'Ivoire, la faisabilité d'un contrôle individuel de performances en milieu villageois était démontrée (42). 
Dans ce contexte, l'Isra-Lnerv* et le Cirad-emvt ont mis en place le programme Pathologie et productivité des petits ruminants en milieu traditionnel au Sénégal (Ppr). Ce projet pluridisciplinaire devait appliquer une approche systémique à l'étude de la productivité des ovins et des caprins en milieu traditionnel, en centrant les observations et les objectifs opérationnels sur l'unité zootechnique et épidémiologie la plus pertinente : le troupeau de concession.

Les objectifs du programme Ppr étaient :

- d'établir un référentiel socio-économique, zootechnique et sanitaire sur l'élevage des ovins et des caprins en milieu traditionnel ; - d'étudier les facteurs de variations de ces paramètres ;

- de proposer des thèmes d'amélioration adaptés aux stratégies, aux contraintes de production et aux objectifs économiques des éleveurs (49).

En préliminaire aux études proprement dites, il était nécessaire de mettre au point un système fiable de collecte et de contrôle des informations zootechniques et sanitaires individuelles. Ce travail méthodologique a été réalisé de 1983 à 1985 et a abouti au système d'information Panurge (10), utilisé pendant 13 ans. Ce système a été repris par d'autres projets de recherche ou de développement au Sénégal et dans d'autres pays. Un ensemble de jeux de données a été constitué, couvrant des situations agro-écologiques et climatiques variées et recueillies selon un protocole identique.

Par sa conception, l'outil informatique était orienté vers la collecte et le contrôle des données et non vers leur traitement. Il ne permettait que des requêtes limitées, sans possibilité d'agrégation ni de relations entre tables. L'information d'un même domaine était parfois dispersée dans plusieurs tables. Par exemple, les poids de croissance, les poids post-partum et les poids à l'embouche étaient situés dans trois tables différentes. Pour pallier l'absence de relation entre les tables, des redondances avaient été introduites dans les différents fichiers. Ainsi, le code de troupeau, la date de naissance de l'animal et le numéro d'identification de sa mère étaientils répétés dans la plupart des tables. Cela ouvrait la voie à des erreurs de mise à jour qui sont devenues nombreuses au fil du temps. A titre d'illustration, certains animaux étaient signalés présents dans des troupeaux différents au même moment, ou un même animal avait plusieurs carrières de croissance différentes. L'utilisation de cet outil rendait donc difficiles l'exploitation et la synthèse des données.

Cet article présente la méthodologie employée pour construire une base de données homogène, corrigée et simplifiée, afin de faciliter l'extraction des données selon des points de vue variés. Les principales caractéristiques de cette base de données sont ensuite présentées ainsi que les utilisations actuellement envisagées.

\section{MATERIEL ET METHODES}

\section{Système de collecte des informations de terrain}

Le système d'information Panurge a été présenté de manière détaillée $(10,17)$. Le troupeau de concession, défini comme l'ensemble des animaux passant la nuit dans la même concession, avait été choisi comme l'unité zootechnique, épidémiologique, statistique et opérationnelle. Les travaux fondateurs de Landais et Sissokho et Poivey et coll. $(23,42)$ avaient démontré l'importance

* Institut sénégalais de recherches agricoles - Laboratoire national d'élevage et de recherche vétérinaire de la qualité des données individuelles pour obtenir des paramètres démographiques et zootechniques fiables. Les variables de troupeaux devaient être obtenues par agrégation de données animales individuelles de bonne qualité. A cet effet, des informations zootechniques et sanitaires ont été recueillies à un rythme régulier dans des élevages observatoires sédentaires choisis pour représenter la diversité des conditions agro-écologiques du Sénégal. Dans ces élevages, chaque animal était identifié à l'aide d'une boucle auriculaire plastifiée pré-imprimée.

L'enquête s'articulait autour d'un suivi démographique des troupeaux auquel étaient adjoints divers modules (croissance pondérale, santé, pratiques d'élevage...), variables selon le protocole. Des techniciens spécialement formés et basés sur le terrain effectuaient chaque quinzaine des visites d'élevage pour relever les changements démographiques. Les autres informations étaient recueillies à un rythme adapté au type de données : tous les trois jours pour l'étude des fréquences pathologiques, toutes les semaines ou chaque quinzaine pour le suivi de la production laitière, à chaque saison pour le suivi des pratiques ou tous les ans pour le compte d'exploitation. Ces techniciens tenaient à jour un fichier manuel et transmettaient régulièrement les données au laboratoire. La saisie des données était effectuée à l'aide d'une application spécifiquement développée avec un système de gestion de base de données non relationnelles (11). De nombreux contrôles de cohérence étaient automatiquement effectués au moment de la saisie des informations. Les données rejetées par le logiciel étaient retournées sur le terrain où elles étaient vérifiées et corrigées.

\section{Conception de la base de données}

Une publication récente (49) a présenté les principales études réalisées par le programme Ppr. Il n'était pas utile de reprendre la totalité des informations recueillies depuis 1983 : certaines données ont été exploitées (évolution de la dentition avec l'âge (45)), ou ne présentaient qu'une faible variabilité (prévalence parasitologique des hémoparasitoses). Par ailleurs, il était nécessaire d'organiser la base de données pour permettre une utilisation facile dans quatre domaines prioritaires :

- la mise à jour des référentiels zootechniques de productions ovine et caprine ;

- la dynamique des populations de petits ruminants à l'échelle du troupeau ;

- les relations entre production et pathologie ;

- l'estimation des paramètres génétiques des caractères quantitatifs.

La liste des informations nécessaires a été obtenue en examinant les données disponibles sous forme de fichier électronique ou papier, en consultant les utilisateurs actuels et futurs de la base de données et en comparant avec les informations figurant dans d'autres bases de données zootechniques ou épidémiologiques (4, 20, 24, 27, 28, 41, 42, 46, 47, 52).

Les différents niveaux d'agrégation et échelles temporelles ont été définis selon les besoins des utilisateurs de la base de données. Un modèle des données a ensuite été établi en utilisant le formalisme graphique de l'approche entité-relation (2). Cette méthode a été développée et standardisée en France sous le nom de Merise (48). Elle a été utilisée pour concevoir des bases de données zootechniques et épidémiologiques $(27,28)$. Elle consiste à identifier des entités et des relations dans l'ensemble formé par les données élémentaires et leurs liens. Le tout conduit à un modèle conceptuel des données (Mcd) exhibant la complexité des relations entre les données. Le Mcd est un ensemble de diagrammes simples que l'on 
peut regrouper par niveau d'agrégation. Ce support graphique permet de présenter la base de données à des non spécialistes en informatique. Des critiques et des amendements peuvent ainsi être apportés par les utilisateurs de la base de données. Un résultat satisfaisant est obtenu en quelques itérations.

Après acceptation du Mcd, la conception technique de la base de données a été effectuée. Le Mcd a été traduit sous une forme physique tenant compte des choix techniques tels que la construction d'un schéma relationnel. Cette étape a nécessité un important travail de contrôle de cohérence et de vérifications. Elle a abouti à un modèle physique des données organisées en tables logiquement reliées entre elles.

\section{RESU LTATS}

\section{Données retenues}

Quatre jeux de données ont été retenus pour constituer la base de données (figure 1) selon des critères de fiabilité des données, d'absence de traitements antérieurs et d'urgence des besoins des chercheurs et des utilisateurs des données.

Les données de Kaolack ont été relevées dans le cadre du Prodec* qui souhaitait connaître l'évolution des paramètres démographiques des troupeaux de petits ruminants encadrés par le projet. Les données de Kaymor, Kolda et Louga ont été recueillies dans le cadre des activités du programme Ppr. Pendant toute la durée de suivi sur ces sites, la démographie des troupeaux et la croissance des jeunes ont été suivies.

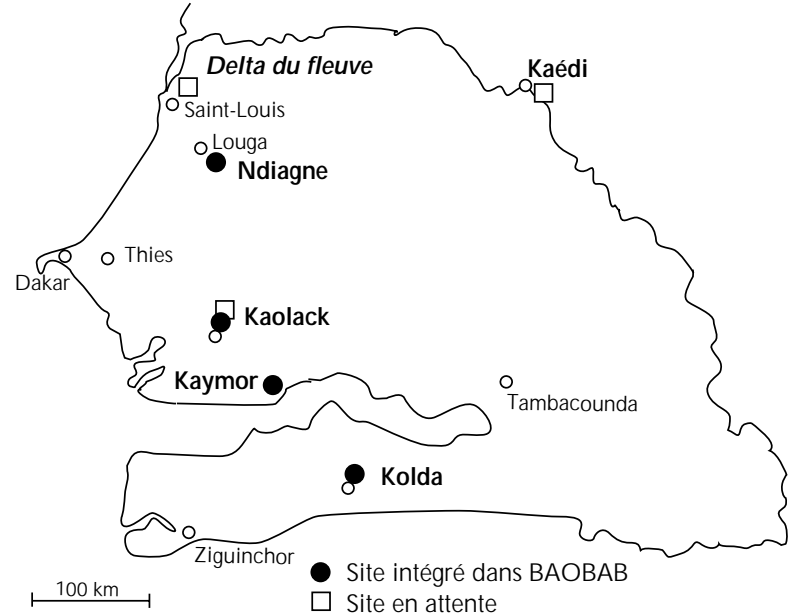

Figure 1 : répartition des sites inclus dans la base de données et des sites candidats.

Au-delà de ces données de base, différentes études ont été réalisés sur les pratiques de conduite et d'exploitation $(8,9,36)$, les relations entre pratiques d'élevage et performances animales (35), la séro-épidémiologie des agents pneumotropes pathogènes (25) et des maladies abortives (37), la dynamique saisonnière des dominantes pathologiques, l'épidémiologie des parasitoses gastro-intestinales, les fréquences pathologiques et les relations entre le statut nutritionnel et les performances zootechniques. Les principales caractéristiques de chaque jeu de données sont présentées dans le tableau I.

* Projet de développement des espèces à cycles courts, Direction de l'élevage, financé par le Fonds d'aide et de coopération, France

Tableau I

Domaines et périodes concernés dans les jeux de données incorporés dans la base de données Baobab

\begin{tabular}{|c|c|c|c|c|}
\hline \multirow[t]{2}{*}{ Domaines } & \multicolumn{4}{|c|}{ Périodes } \\
\hline & Kaolack & Kaymor & Kolda & Louga \\
\hline D émographie & 05/1995-06/1997 & 04/1984-07/1994 & 12/1983-03/1998 & 06/1983-08/1997 \\
\hline Croissance & 05/1995-06/1997 & 04/1984-07/1994 & 12/1983-03/1998 & 06/1983-08/1997 \\
\hline $\begin{array}{l}\text { Pratiques d'élevage } \\
\text { (logement, alimentation } \\
\text { complémentaire, traite, } \\
\text { conduite au pâturage) }\end{array}$ & - & SP/1988-SS/1993 & SP/1989-SS/1994 & SP/1988-SS/1994 \\
\hline Embouche ovine & 05/1995-03/1997 & 12/1989-06/1994 & - & 12/1988-07/1997 \\
\hline $\begin{array}{l}\text { Essais de schémas } \\
\text { prophylactiques }\end{array}$ & - & 09/1984-11/1992 & 06/1984-12/1992 & 10/1983-09/1992 \\
\hline $\begin{array}{l}\text { Dynamique des dominantes } \\
\text { pathologiques }\end{array}$ & - & 12/1987-04/1994 & 10/1987-04/1996 & 01/1988-04/1996 \\
\hline Fréquences pathologiques & - & 03/1991-04/1994 & 02/1991-03/1995 & 02/1991-03/1996 \\
\hline $\begin{array}{l}\text { Epidémiologie des agents } \\
\text { pathogènes respiratoires }\end{array}$ & - & - & 12/1987-06/1989 & $11 / 1987-05 / 1989$ \\
\hline $\begin{array}{l}\text { Epidémiologie } \\
\text { des maladies abortives }\end{array}$ & - & - & 10/1993-04/1994 & 10/1993-04/1994 \\
\hline $\begin{array}{l}\text { Epidémiologie des parasitoses } \\
\text { gastro-intestinales }\end{array}$ & - & 11/1992-12/1993 & 08/1992-11/1995 & 09/1992-01/1994 \\
\hline $\begin{array}{l}\text { Statut nutritionnel } \\
\text { (profils biochimiques) }\end{array}$ & - & - & 10/1993-04/1994 & 10/1993-04/1994 \\
\hline M étéorologie & 05/1995-06/1997 & 04/1984-07/1994 & 12/1983-03/1998 & 06/1983-08/1997 \\
\hline
\end{tabular}

SP : saison pluvieuse ; SS : saison sèche 


\section{Modèle conceptuel des données}

L'animal et le troupeau de concession étaient les deux principaux niveaux d'agrégation. La localisation géographique (spatialisation des données) et le temps chronologique (variations saisonnières et annuelles) devaient permettre de définir d'autres niveaux d'analyse. Pour faciliter les analyses, le temps chronologique a été exprimé sous forme de dates.

Le rang de mise bas et le stade de lactation sont des éléments essentiels pour les analyses touchant à la carrière reproductrice des femelles ou aux cycles de production individuels (productivité numérique et pondérale, production laitière). Deux échelles de temps physiologique ont été définies : le rang de mise bas et la semaine de lactation (nombre de semaines écoulées entre la dernière mise bas et la date considérée).

Un troisième rythme était susceptible d'avoir une influence sur les données : le temps météorologique. Les données ont été fournies par la Météorologie nationale du Sénégal et incorporées dans la base sous forme décadaire.

Le Mcd a été élaboré en une dizaine de jours. Il a été validé au cours de visites de terrain et d'une réunion générale de tous les intervenants (figures 2 et 3 ).

\section{La base de données Baobab}

La traduction de ce Mcd sous forme physique a conduit à une structure de base de données comportant 28 tables (tableau II). Un exemple permet d'illustrer le passage du Mcd à la base de données. La relation « croissance » reliant les entités ANIMAL, MESURE et DATE (figure 2) a donné la table DateMesu (tableau II). Cette table comporte les champs «numani » (numéro d'identification de l'animal : clé de l'entité ANIMAL), « date » (date de la mesure : clé de l'entité DATE), «mesure » (type de mesure effectuée : clé de l'entité MESURE). Dans cette table, la combinaison de ces trois champs est unique : il ne doit pas y avoir deux enregistrements avec le même numéro d'identification, la même date et le même type de mesure. Ceci est une règle de gestion permettant d'effectuer des contrôles sur la table (recherche de doublons). Les autres champs de la table DateMesu sont les propriétés de la relation « croissance »: les champs « calcobs » (mesure observée ou calculée) et «valeur» (valeur de la mesure).

Des programmes de codage et de contrôle ont été écrits pour saisir les informations manquantes et transférer les données vers la nouvelle structure. Panurge n'étant pas une base de données relationnelles, de nombreuses redondances et erreurs de mise à jour ont été rencontrées. Le transfert a nécessité l'écriture de 173 contrôles

\section{Tableau II}

Description qualitative du contenu des tables de la base de données Baobab

\section{Table Description}

Absent Liste des animaux absents à chaque visite. Nécessaire pour exploiter Patho15

Aliment Valeur alimentaire des aliments utilisés dans les rations d'embouche ovine

AlimPart Périodes de distribution, nature, quantité et prix des aliments constituant les rations d'embouche ovine

Animal Données d'état-civil constantes des animaux (identificateur, mère, père, date de naissance, type génétique...)

Boucle Liste des boucles ou numéros provisoires portés par un animal au cours de sa présence dans le suivi

CompConc Pratiques d'alimentation complémentaire par troupeau de concession, saison, espèce et année

ConcAni Périodes de présence des animaux dans les troupeaux avec caractéristiques des entrées et sorties

Concess Données constantes sur les troupeaux de concession (nom et ethnie de l'éleveur, troupeau ascendant...)

Concloge Types de logement occupés par un troupeau de concession au cours des saisons et des années

ConcPatu Pratiques de pâturage : types et périodes de fréquentation des pâturages par troupeau, espèce, saison et année

CondG ene Modes de conduite au pâturage et pratiques de traite par troupeau de concession, espèce, saison et année

CondPart conduite particulière (ex. : mise à l'embouche) des animaux qui ne suivent pas la conduite générale sur parcours

DateM esu Poids vifs et notes d'état corporel relevées sur les animaux en suivi

Fraicond Frais induits par une conduite particulière (embouche) sur un lot d'animaux d'un troupeau pendant une période donnée

InterAni Interventions subies par animal (traitement, prophylaxie, castration). Les interventions collectives sont dans InterCol

InterCol Interventions collectives (vaccin, vermifuge) réalisées à l'échelle d'un village ou d'un troupeau de concession

Labo

Lieu Résultats d'analyses de laboratoire individuelles (analyses biochimiques, sérologies, coproscopies...)

Meteo Données constantes par village : nom, coordonnées géographiques, station météorologique...

Données climatologiques par station météorologique et par décade

Misebas Informations relatives à la mise bas et à ses caractéristiques en termes de productivité numérique et pondérale

Mouvment Périodes de suivi et localisation des troupeaux de concession (débuts et fins de suivi, déplacements, transhumance)

Patho Diagnostics, gravité, durée des pathologies observées lors de l'étude des fréquences pathologiques

Patho15 Symptômes respiratoires et digestifs relevés chaque quinzaine lors de l'étude sur les dominantes pathologiques

ProtoSer Description des séries de prélèvements réalisés et éléments recherchés pour chaque protocole d'analyse

SeM esu Poids vifs et notes d'état corporel des femelles après la mise bas, selon la parité et la semaine de lactation

SemLait Prélèvements laitiers des éleveurs sur les femelles en lactation, selon la parité et la semaine de lactation

Symptome Symptômes par appareil observés lors de l'étude des fréquences pathologiques

Transact Informations concernant les achats et les ventes d'animaux 


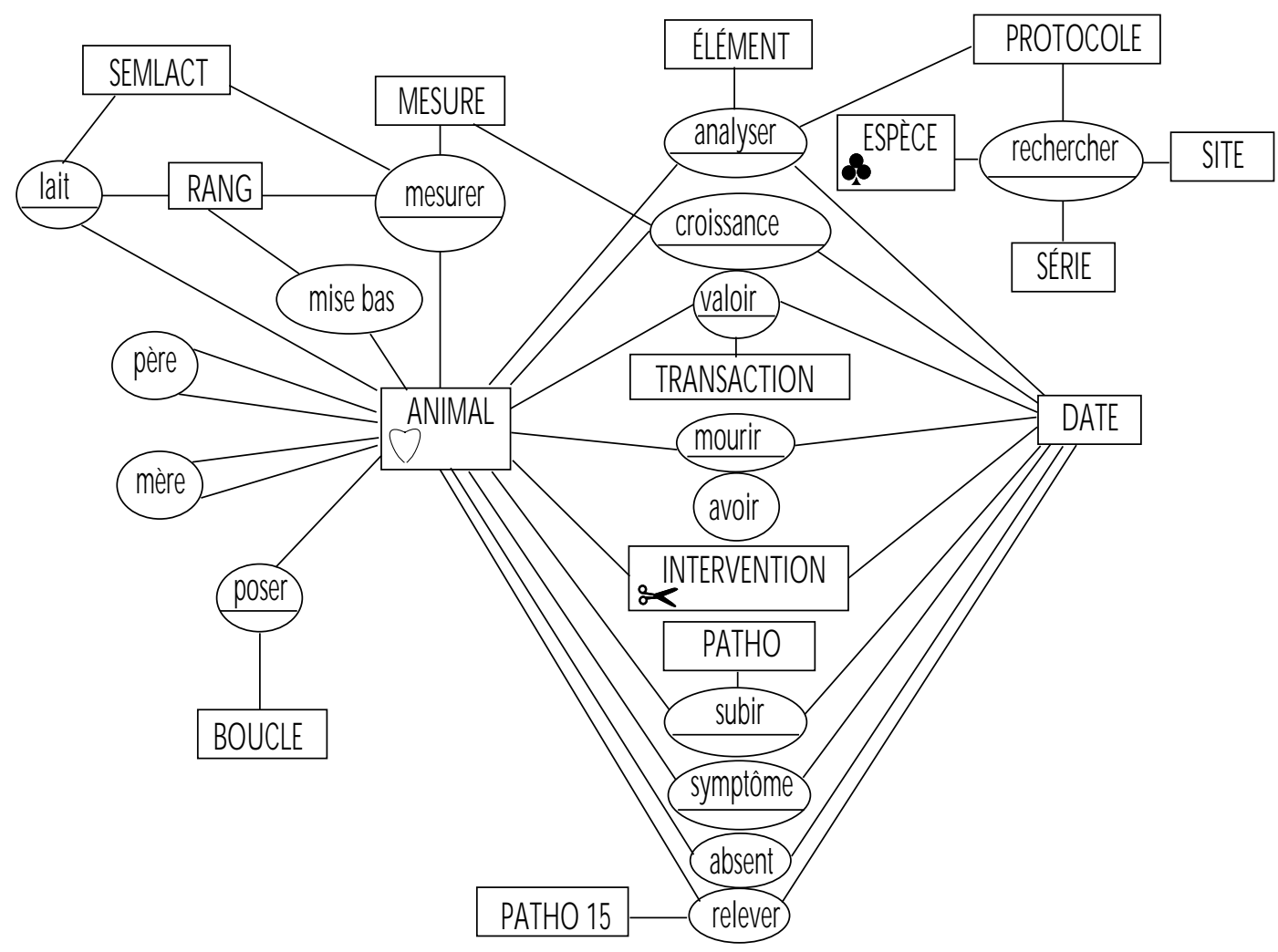

Figure 2 : modèle conceptuel des données. I. Données individuelles. Les relations sont figurées par des ellipses, le trait horizontal éventuel indiquant la présence de propriétés. Les entités sont représentées par des rectangles. Toutes les entités ont au moins une propriété : Ia clé. Les symboles en bas à gauche de certaines entités indiquent que ces dernières sont retrouvées en plusieurs endroits du Mcd.

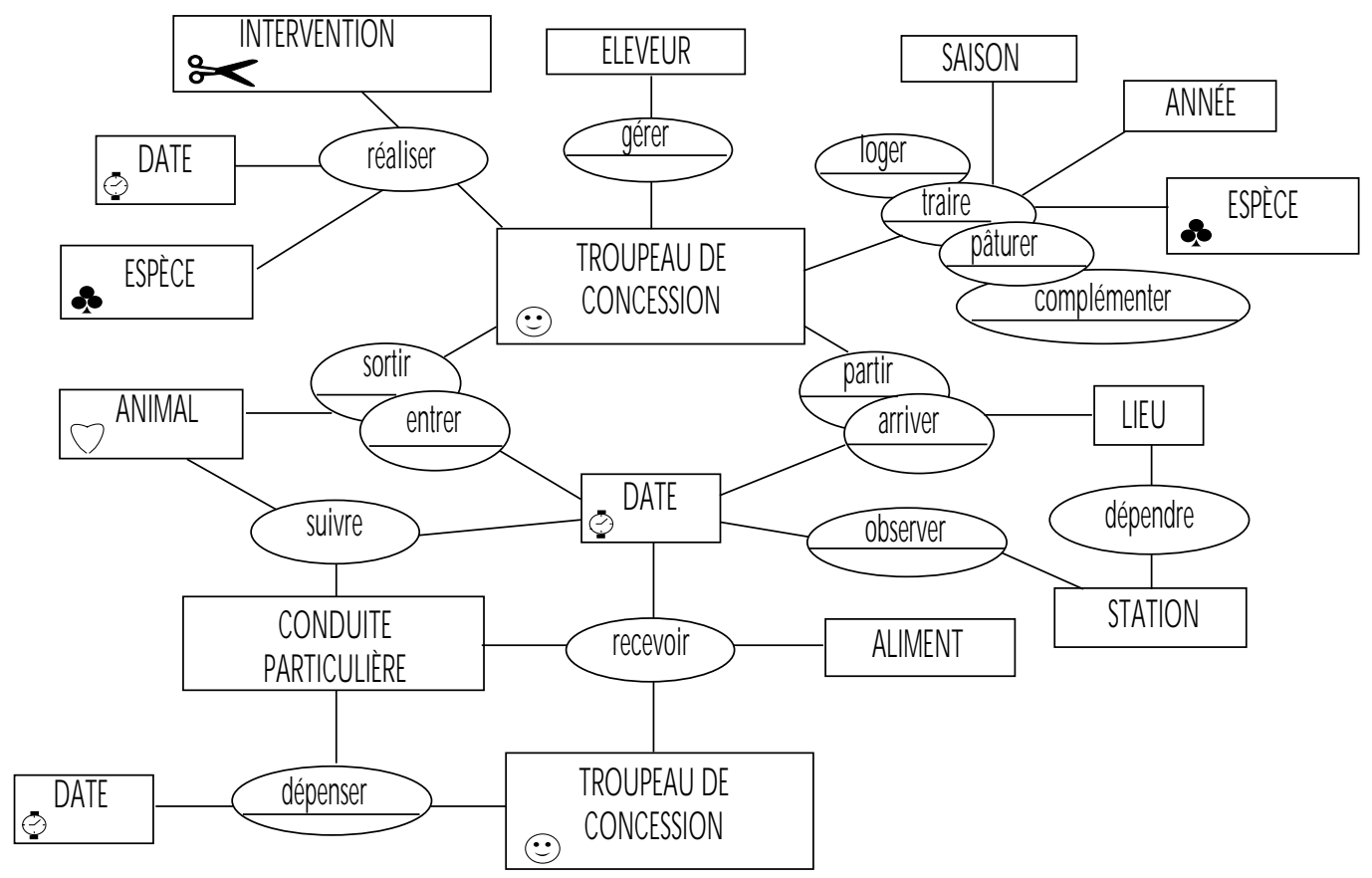

Figure 3 : modèle conceptuel des données. II. Représentation des relations entre l'animal et le troupeau de concession et des données relatives au troupeau de concession. Les relations sont figurées par des ellipses, le trait horizontal éventuel indiquant la présence de propriétés. Les entités sont représentées par des rectangles. Toutes les entités ont au moins une propriété : la clé. Les symboles en bas à gauche de certaines entités indiquent que ces dernières sont retrouvées en plusieurs endroits du Mcd. 
pour vérifier et corriger les données. La phase de saisie et de correction a duré plus d'un an et a mobilisé à temps plein une équipe composée d'un chercheur administrateur de la base de données et de deux techniciens. La base de données a été implantée sur micro-ordinateur à l'aide d'un système de gestion de base de données relationnelles (Sgbdr). Elle occupe un espace d'environ 50 méga-octets avec le Sgbdr utilisé. Compte tenu de sa taille, de la lenteur de sa croissance et de sa localisation géographique, la base de données a été appelée Baobab (base d'observation des animaux qui broutent), à l'image de l'arbre Adansonia digitata, symbole du Sénégal.

Le tableau III donne quelques indications sur la quantité des données disponibles dans Baobab. Les figures 4 à 6 présentent la distribution de quelques variables qui seront souvent utilisées dans le dépouillement de la base de données.

Les Sgbdr utilisés pour la gestion de Baobab (Visual FoxPro* ou Access**) possèdent des langages de gestion des données évolués, permettant d'extraire facilement les informations désirées. Ils offrent en particulier des interfaces sql (structured query language) permettant d'écrire des requêtes peu dépendantes du Sgbdr : un utilisateur non familiarisé avec les langages d'un Sgbdr particulier pourrait travailler rapidement avec Baobab. Le tableau IV donne un exemple de requête sql pour le calcul des fréquences des mises bas ainsi que les premières lignes du tableau obtenu. Le graphe de densité de probabilité de mise bas selon le jour de l'année est un exemple d'utilisation de ce tableau.

* Microsoft Visual FoxPro, version 3.0, système de gestion de base de données relationnelles pour Windows, Microsoft Corporation, 1995

** Microsoft Access, version 7.0, système de gestion de base de données relationnelles pour Windows, Microsoft Corporation, 1989-1997
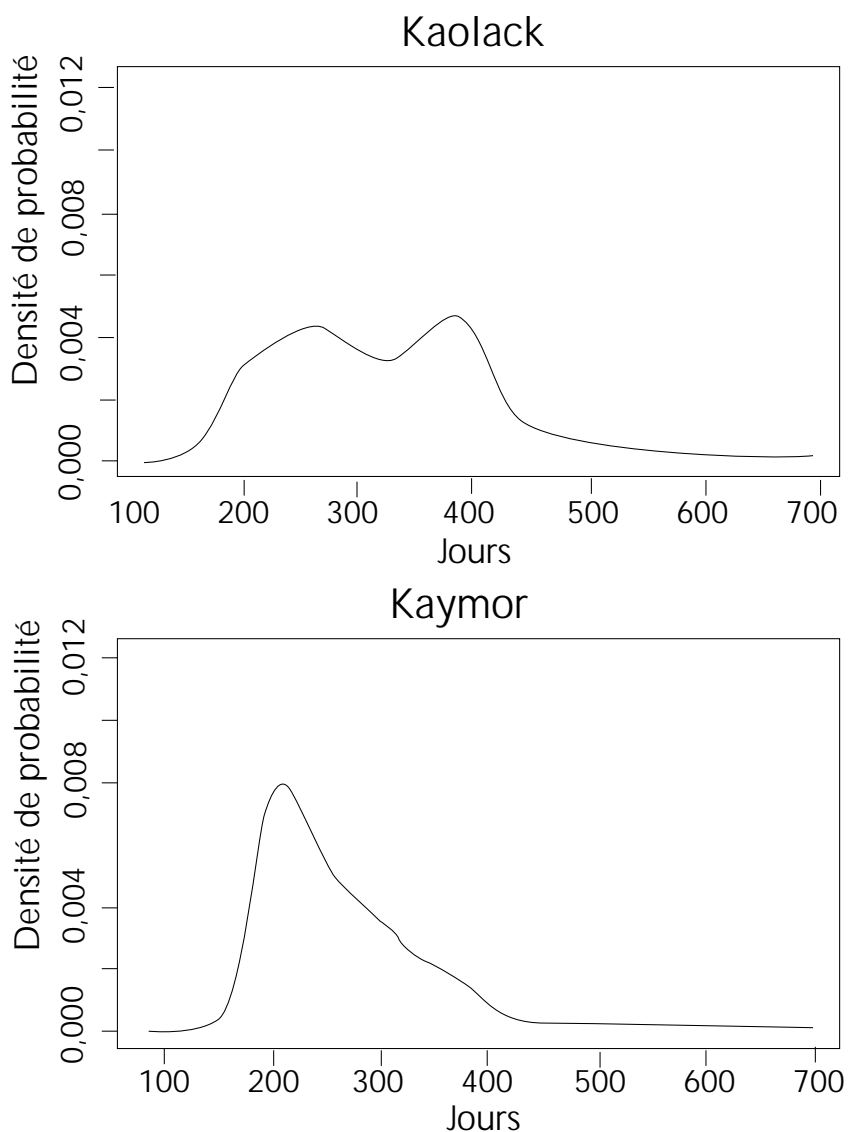

$$
n=23775 \text { intervalles entre mises bas }
$$

La complexité et la diversité des données figurant dans Baobab ont entraîné la nécessité d'écrire deux documents d'accompagnement. Le premier donne une description détaillée de la base de données ainsi que la signification des codes utilisés. Le second rassemble les différents protocoles avec lesquels les données ont été recueillies. Ces documents sont indispensables à une utilisation efficace des données.
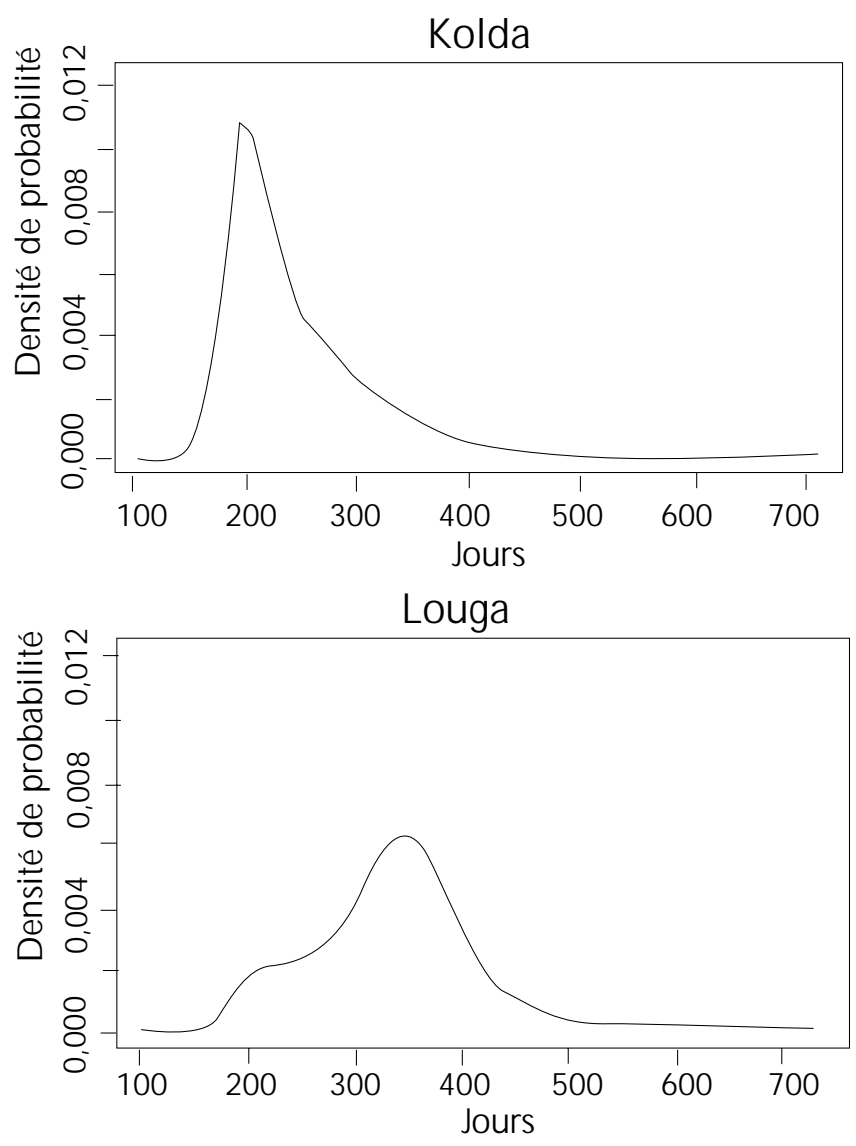

Figure 5 : distribution des intervalles entre mises bas normales successives chez la brebis ( $n=23775$ intervalles entre mises bas). 


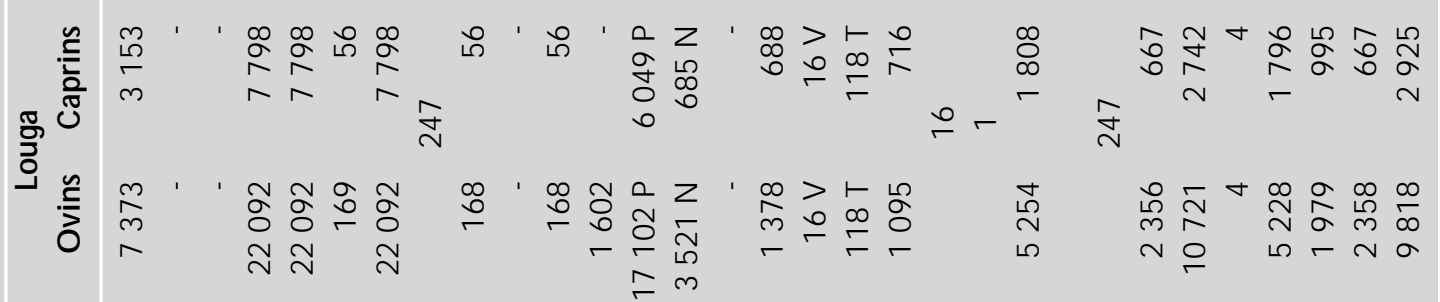

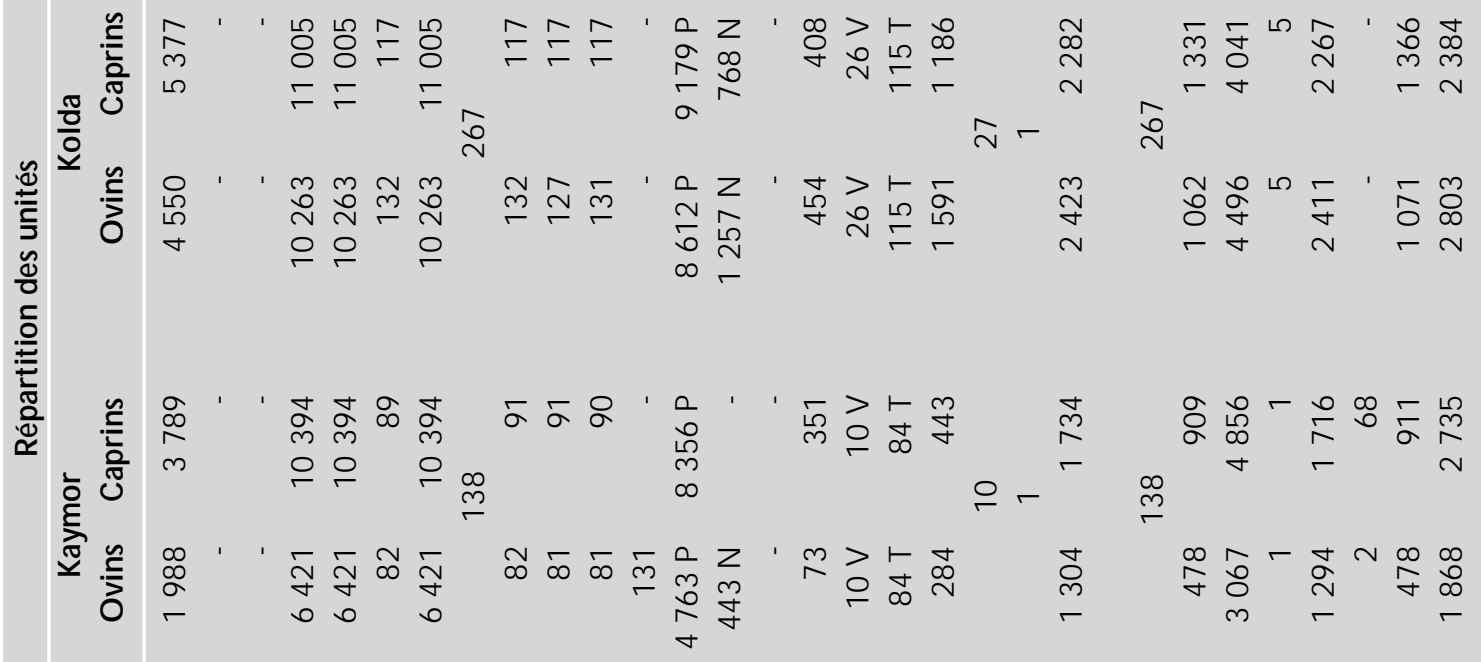

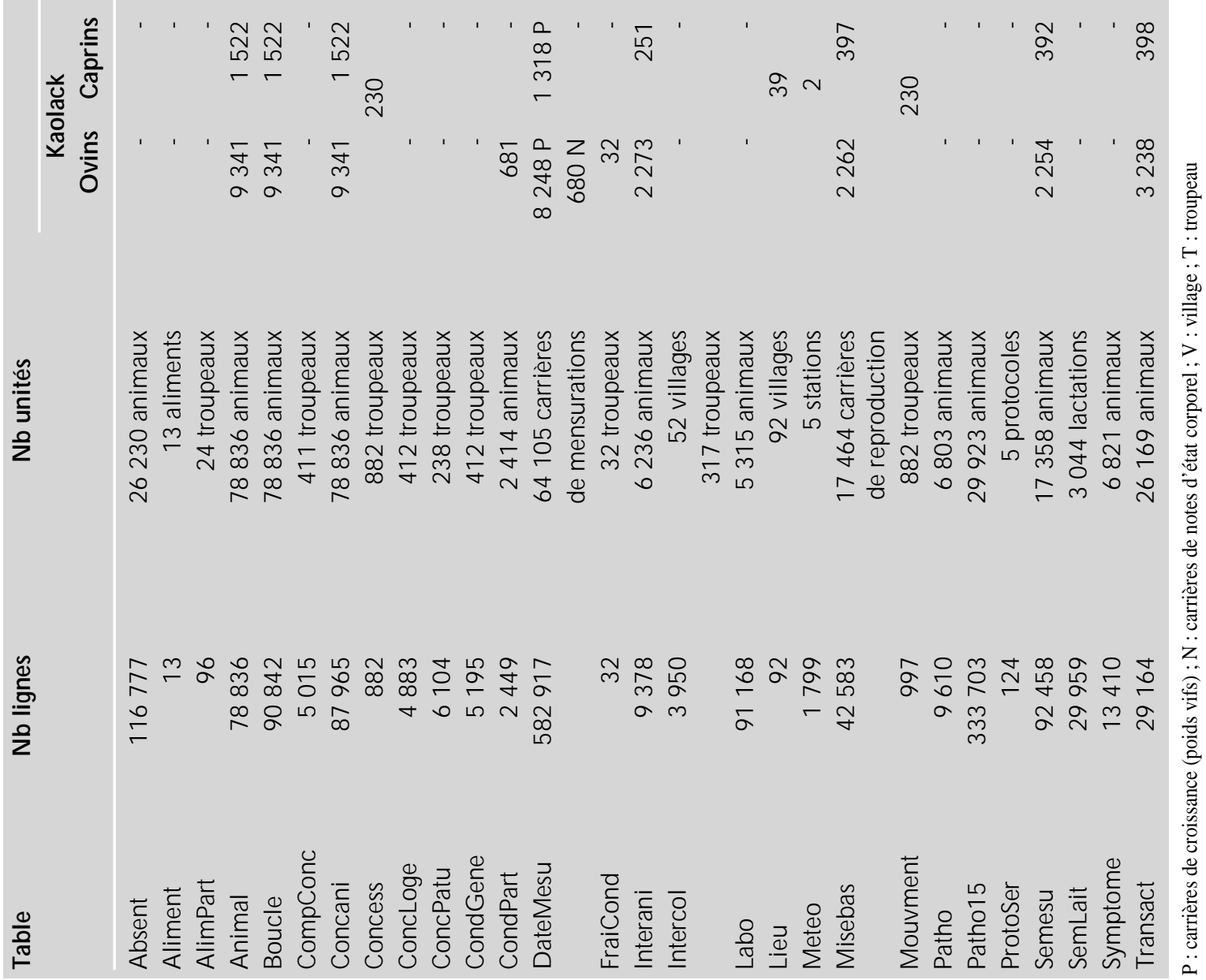




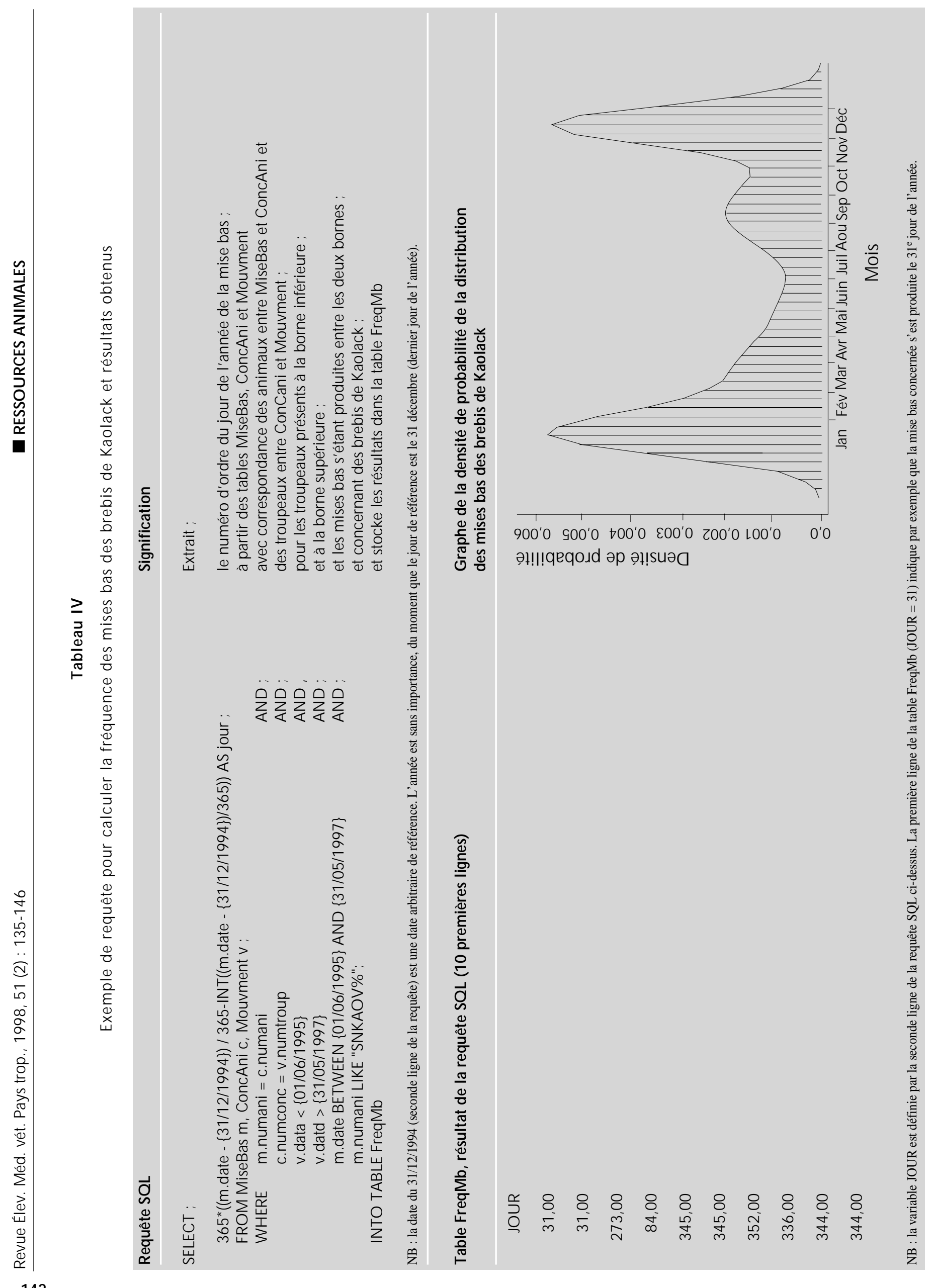




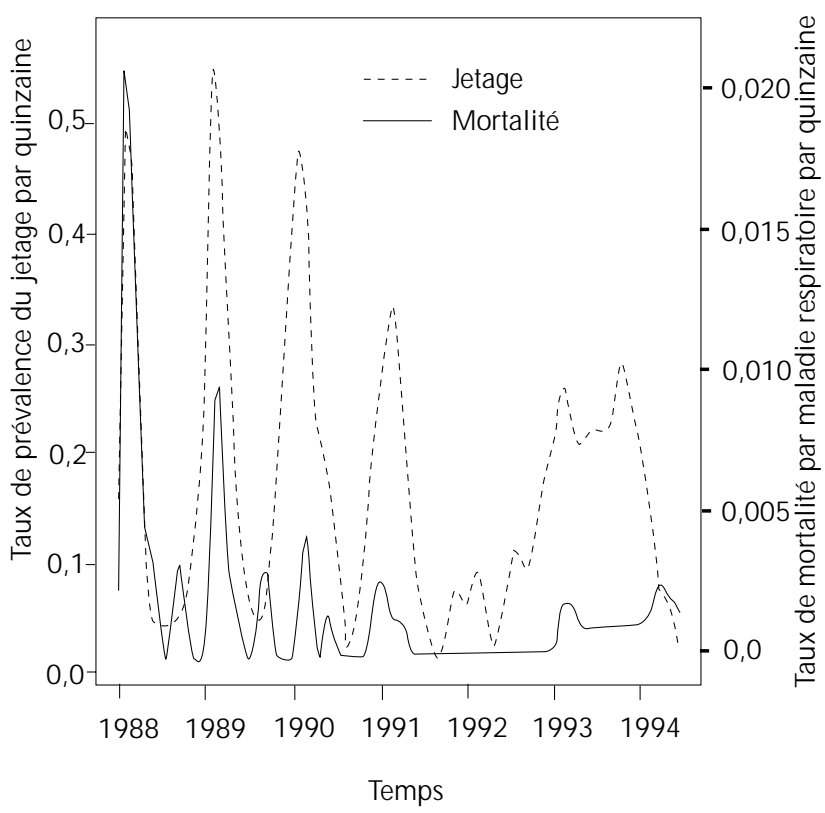

Figure 6 : prévalence du jetage et taux de mortalité par maladie respiratoire chez les ovins à Kolda.

\section{DISCUSSIO N}

\section{Données retenues et données en attente}

La base présentée peut être complétée. Les tables concernant le troupeau de pâturage (ensemble des troupeaux de concession rassemblés pour aller au pâturage) n'ont pas été incluses dans Baobab. Des informations sur le berger, la nature des pâturages fréquentés et leur charge animale, les espèces végétales et la digestibilité de la ration consommée sur une période d'un an à Louga (juillet 1996 - juin 1997) ont été recueillies à l'occasion d'un protocole d'étude des relations entre les ressources fourragères naturelles et la productivité animale. Les tables seront ajoutées dans Baobab à l'occasion de l'analyse de ces données. D'autre part, des jeux complets de données attendent d'être incorporés dans Baobab (figure 1) : suivis du delta et de la moyenne vallée du fleuve Sénégal (Saint-Louis $(50,51)$ et Kaédi en Mauritanie (14)), première enquête de Kaolack (31). Il serait également possible d'inclure les données de bovins suivis à Kolda ou Kaymor dans le cadre d'autres programmes de recherche ayant utilisé Panurge. D'importantes bases de données ont été constituées sur les bovins au Burkina Faso et en Nouvelle-Calédonie $(32,33)$, et sur les ovins en Côte d'Ivoire (5) avec le même système d'information.

L'intérêt d'accumuler de nombreux jeux de données récoltés selon des protocoles identiques dans des zones agro-écologiques proches est double. Tout d'abord, c'est l'occasion de valider des modèles établis sur un jeu de données particulier et de vérifier leur caractère général. Par exemple, les animaux du suivi de Saint-Louis (delta du fleuve) ont subi pendant l'hivernage de 1987 une épidémie de fièvre de la vallée du Rift (21). Cette zoonose a provoqué chez les ruminants des taux d'avortement et une mortalité néonatale très élevés. On pourrait ainsi étudier le comportement de modèles de dynamique de population devant des événements catastrophiques. Ensuite, il est possible de réaliser des méta-analyses $(15,19)$, c'est-à-dire d'examiner la variabilité des résultats d'une même expérimentation (protocole vaccinal, calendrier de vermifugation, alimentation complémentaire...) répétée dans des conditions et dans des endroits différents, afin d'obtenir une meilleure estimation des effets étudiés.

\section{Conception de la base de données et retombées}

Le Mcd retenu pour Baobab est original. Il s'appuie sur des recommandations générales sur les données zootechniques devant figurer dans une base de données (44) et la manière de les modéliser (27). Il est d'une complexité analogue ou supérieure à celle d'autres bases de données zootechniques et sanitaires constituées dans des élevages bovins laitiers intensifs, commerciaux $(26,27)$ ou expérimentaux $(4,28)$. Il est en particulier adapté à la gestion des mouvements d'animaux entre troupeaux, phénomène rare dans les systèmes intensifs d'Europe mais très fréquent dans les élevages sénégalais étudiés.

La mise au point du Mcd de Baobab et le travail de transfert des données ont mis en évidence les faiblesses de conception informatique des logiciels de saisie utilisés jusqu'alors. Les travaux de comparaison des résultats d'enquêtes longitudinales et transversales montrent qu'il est difficile de se passer de données individuelles précises et fiables dès que sont abordés les problèmes de dynamique de population $(23,29,53)$. En conséquence, un nouveau logiciel de saisie de données individuelles a été écrit, reposant sur un Mcd simplifié de Baobab. Cette application, dénommée Laser (logiciel d'aide au suivi des élevages de ruminants), comporte 11 tables principales, équivalentes aux tables Animal, Boucle, ConcAni, Concess, DateMesu, InterAni, InterCol, Lieu, MiseBas, Mouvment et Transact de Baobab. Laser peut gérer des mouvements de troupeaux à l'occasion des transhumances ou de déplacements conjoncturels (sécheresse, épidémie...). Les lieux de séjour des troupeaux sont repérés par des coordonnées géographiques. Une interface est possible avec un système d'information géographique, permettant de prendre en compte les structures spatiales sous-jacentes telles que les ressources fourragères ou les facteurs édaphiques. L'application a été écrite à l'aide d'un langage de programmation courant (Visual Basic*) avec un cahier des charges précis. Cela assure la possibilité de maintenance et d'évolution en fonction des besoins des utilisateurs. Laser alimente une base de données au format Access, largement répandu, ce qui facilite les transferts vers d'autres Sgbdr et la plupart des logiciels de statistiques actuels.

Cependant, le logiciel de saisie est une partie mineure de l'ensemble du système d'information. Le point essentiel reste la qualité du réseau d'observation, constitué par des éleveurs partenaires et bénéficiaires (dans un schéma de recherche - action) de l'enquête, des observateurs correctement formés et un protocole précis et rigoureux de collecte et de contrôle des données. En cela, le système d'information Panurge (10) est et restera longtemps d'actualité, même si l'outil informatique initial est remplacé par un outil plus moderne.

\section{Exploitation de la base de données et perspectives d'utilisation}

L'aspect longitudinal des données de croissance (figure 4) a été peu exploité en dehors des travaux de Moulin (35) qui a établi des profils de croissance et les a reliés à des pratiques d'élevage pour les petits ruminants de Ndiagne (zone sahélienne). Les intervalles entre mises bas (figure 5) et les autres paramètres de reproduction ont été présentés $(3,18)$. Clément et coll. (3) ont mis l'accent sur

* Microsoft Visual Basic, version 5.0, système de programmation pour Windows 95 et NT, Microsoft Corporation, 1991-1997 
l'aspect génétique (héritabilités et corrélations génétiques) mais les carrières reproductrices n'ont été étudiées que chez les brebis et les chèvres de Ndiagne (35). Les relations entre les symptômes respiratoires et les séroconversions envers les germes pneumotropes ont été étudiées par Lefèvre-Desoutter (25) à Ndiagne et à Kolda (zone soudano-guinéenne), mais l'impact de la pathologie respiratoire sur la productivité (figure 6) n'a pas été quantifié. L'épidémiologie des parasitoses digestives a fait l'objet de plusieurs études mais rien n'a été publié jusqu'à présent.

A travers ces exemples, il apparaît que les perspectives d'utilisation de la base de données sont nombreuses. La mise à jour des référentiels zootechniques de productions ovine et caprine publiés en 1991 (18) est nécessaire. Elle se fera à travers des articles synthétiques publiés dans des revues largement diffusées en milieu tropical. Les résultats seront ainsi disponibles pour les comparaisons. La revue récente de de Leeuw et coll. (24) sur le suivi de la santé et de la production du bétail en Afrique sub-saharienne a montré la faible diffusion des résultats du Sénégal, en contradiction frappante avec la qualité et la quantité des données récoltées. Sur les 104 articles cités en références, deux émanaient du programme Ppr dont aucun ne présentait de résultats zootechniques complets. D'autre part, des fiches techniques seront établies sur des sujets précis établis en concertation avec la Direction de l'élevage, les services de développement et les organisations de producteurs du Sénégal. A priori, des sujets tels que la vaccination contre les grandes endémies des petits ruminants, la vermifugation contre les parasitoses digestives ou les techniques d'embouche devraient être traités en priorité.

La dynamique des populations de petits ruminants à l'échelle du troupeau fait l'objet d'un travail de thèse de biométrie sur les modèles matriciels en temps discret et l'application à la dynamique des petits ruminants domestiques au Sénégal. L'objectif à court terme est d'établir une méthode de diagnostic démographique d'une population domestique à partir des paramètres de mortalité, de fertilité et d'échanges migratoires. Dans un second temps, il est envisagé de travailler sur les relations entre la démographie et l'état individuel des animaux, en particulier les relations entre la croissance des femelles et la démographie.

La description de la pathologie doit être réalisée préalablement à l'étude des relations entre production et pathologie. Il est nécessaire d'établir l'épidémiologie descriptive des pathologies respiratoire et digestive et de faire le lien avec les résultats de laboratoire disponibles (sérologies des agents respiratoires pneumotropes, coproscopies des parasites digestifs). Des études analytiques seront ensuite entreprises pour préciser l'impact de la pathologie sur la productivité des petits ruminants. La maladie sera considérée comme un facteur de production (facteur de variation d'une variable de production ou de productivité) ou comme la sanction de mauvaises conditions d'élevage (marqueur et conséquence d'un déséquilibre physiologique). Les essais de prophylaxie et de traitement $(12,13)$ pourront être replacés dans cette perspective. Deux échelles différentes seront retenues dans ces analyses. Le niveau de l'animal sera privilégié pour l'étude des carrières (croissance, reproduction, santé et interactions entre ces éléments), afin d'exploiter au mieux l'aspect longitudinal des données. Les analyses à l'échelle du troupeau permettront de dégager les recommandations concrètes à faire aux éleveurs en termes de prophylaxie, d'alimentation complémentaire et de pratiques d'élevage.

Les travaux de génétique quantitative (3) doivent être complétés par l'étude des paramètres de croissance. L'incorporation de nouveaux jeux de données dans Baobab permettra d'augmenter la précision des estimations des valeurs des héritabilités et des corrélations génétiques. L'enjeu de ces travaux est double. Sur le plan méthodologique, il est important de préciser l'intérêt et les limites des données recueillies en milieu villageois. De multiples facteurs de variations et la méconnaissance de l'ascendance paternelle compliquent en effet le calcul des paramètres génétiques. Les premiers résultats montrent que certaines de ces difficultés peuvent être surmontées (3). Une synthèse complète pourrait donner des indications utiles sur les modalités de l'amélioration génétique des petits ruminants. Ce thème intéresse beaucoup les producteurs ovins, très demandeurs d'animaux plus performants (conformation, résistance aux maladies), et la Direction de l'élevage du Sénégal.

\section{CONCLUSION}

La construction de la base de données Baobab a permis de capitaliser les travaux de recherche sur les petits ruminants en milieu traditionnel au Sénégal. Les données ont été triées, homogénéisées et rendues accessibles aux chercheurs de chaque discipline. La maintenance de l'outil est cependant une tâche délicate. Elle nécessite la disponibilité d'un administrateur ayant la responsabilité d'effectuer les corrections et ajouts demandés par les utilisateurs. A ce propos, il faut bien distinguer la base de données Baobab et l'application de saisie Laser. Si Baobab est un outil de recherche complexe et de maintenance délicate, tout a été mis en œuvre pour que l'utilisation de Laser et l'exploitation des bases de données qui en découleront soient aussi simples que possible. L'objectif est de permettre à l'utilisateur de se consacrer aux aspects importants de la recherche en milieu paysan : constitution et gestion du réseau d'observation, exploitation des données et valorisation des résultats.

Les perspectives d'utilisation de la base de données Baobab sont nombreuses et s'orientent selon deux axes principaux :

- la production de référentiels et de synthèses permettant les comparaisons entre pays, zones agro-écologiques et périodes annuelles ; - la modélisation de la production animale, à l'échelle individuelle ou du troupeau.

\section{Remerciements}

La Direction de l'élevage a soutenu les travaux du programme Ppr, en mettant à sa disposition des agents techniques compétents et en orientant les recherches vers ses centres de préoccupation. Les enquêteurs ont permis le recueil des données sur le terrain, avec la collaboration patiente des éleveurs sénégalais. Ils ont également apporté des critiques constructives au Mcd de Baobab. Par ailleurs les auteurs remercient Charles-Henri Moulin, enseignantchercheur à l'Ecole nationale supérieure d'agronomie de Montpellier et précédent responsable du programme Ppr, pour sa contribution particulière au travail de réflexion sur Baobab, ainsi que les trois lecteurs de cet article pour leurs commentaires qui ont contribué à l'amélioration de ce texte.

\section{BIBLIO GRAPHIE}

1. AMEGEE Y., 1983. La prolificité du mouton Djallonké en milieu villageois au Togo. Revue Elev. Méd. vét. Pays trop., 36 : 85-90.

2. CHEN P.P., 1976. The entity-relationship model. Toward a unified view of data. ACM Trans. Database Sys., 1: 9-36.

3. CLEMENT V., POIVEY J.-P., FAU GERE O., TILLARD E., LANCELOT R., GUEYE A., RICHARD D., BIBE B., 1997. Etude de la variabilité des caractères de reproduction chez les petits ruminants en milieu d'élevage traditionnel au Sénégal. Revue Elev. Méd. vét. Pays trop., 50 : 235-249. 
4. COULON J.-B., LESCOURRET F., FAYE B., LANDAIS E., TRO CCO N J.-L. PERO CHON L., 1993. Description de la base de données Lascar, un outil pour l'étude des carrières des vaches laitières. Inra Prod. Anim., 6 : 151-160.

5. DOMENECH J., FORMENTY P., OUATTARA M., N'DEPO A.E., LEFO RBAN Y., 1994. Impacts of major ovine diseases in sub-humid and humid Africa. Kenya Vet., 18: 124-126.

6. FALL A., DIOP M., SANDFORD J., GUEYE E., WISSO CQ Y.J., DURKIN J., TRAIL J.C.M., 1983. Etude sur la productivité de moutons Djallonké au Centre de Recherches zootechniques de Kolda au Sénégal. 1 . Paramètres de reproduction et viabilité. Revue Elev. Méd. vét. Pays trop., 36 : 183-190.

7. FALL A., DIOP M., SANDFORD J., GUEYE E., WISSO CQ Y.J., DURKIN J., TRAIL J.C.M., 1983. Etude sur la productivité de moutons Djallonké au Centre de Recherches zootechniques de Kolda, au Sénégal. 2. Poids corporels, productivité des brebis et du troupeau. Revue Elev. Méd. vét. Pays trop., 36 : 283-289.

8. FAU GERE O., DOCKES A.C., PERROT C., FAU GERE B., 1990. L'élevage traditionnel des petits ruminants au Sénégal. I. Pratiques de conduite et d'exploitation des animaux chez les éleveurs de la région de Kolda. Revue Elev. Méd. vét. Pays trop., 43 : 249-259.

9. FAU GERE O., DOCKES A.C., PERROT C., FAU GERE B., 1990. L'élevage traditionnel des petits ruminants au Sénégal. II. Pratiques de conduite et d'exploitation des animaux chez les éleveurs de la région de Louga. Revue Elev. Méd. vét. Pays trop., 43 : 261-273.

10. FAU GERE O., FAU GERE B., 1986. Suivi de troupeaux et contrôle des performances individuelles des petits ruminants en milieu traditionnel africain. Aspects méthodologiques. Revue Elev. Méd. vét. Pays trop., $39: 29-40$

11. FAU GERE O., FAUGERE B., 1993. Panurge : suivi individuel dans les systèmes d'élevage traditionnel. Maisons-Alfort, France, Cirad-emvt, 339 p.

12. FAU GERE O., LEFO RBAN Y., NERCY C., NDIAYE M., 1987. Essai de traitement des affections respiratoires des petits ruminants du SinéSaloum (Sénégal) à l'aide d'une oxytétracycline à longue action. Revue Elev. Méd. vét. Pays trop., $40:$ 21-32.

13. FAU GERE O., TILLARD E., FAU GERE B., 1992. Prophylaxie chez les petits ruminants au Sénégal : régionalisation d'une politique nationale de protection sanitaire. In: $1^{\mathrm{e}}$ Conf. bienn. Réseau afr. Rech. petits Ruminants, Nairobi, Kenya, 10-15 décembre 1990. Nairobi, Kenya, ILCA, p. 307-315.

14. FRANÇOIS J.-L., 1990. Résultats du suivi zootechnique des petits ruminants : projet d'encadrement des éleveurs du Gorgol (M auritanie). Dakar-Hann, Sénégal, Lnerv, $160 \mathrm{p}$.

15. FRIEDENREICH C.M., 1993. Methods for pooled analyses of epidemiologic studies. Epidemiology, 4: 295-302.

16. HAU MESSER J.B., GERBALDI P., 1980. O bservations sur la reproduction et l'élevage du mouton O udah nigérien. Revue Elev. Méd. vét. Pays trop., $33:$ 205-213.

17. lemvt-Cirad, 1990. Enquête sur la productivité du cheptel : approche pluridisciplinaire basée sur le suivi individuel des animaux. MaisonsAlfort, France, lemvt, 10 p. (Fiche technique d'élevage tropical $n^{\circ} 11$ )

18. lemvt-Cirad, 1991. Enquête par suivi individuel du cheptel. II. Exemples de résultats obtenus sur petits ruminants au Sénégal. MaisonsAlfort, France, lemvt, 8 p. (Fiche technique d'élevage tropical $n^{\circ} 4$ )

19. JONES D.R., 1992. Meta-analysis of observational epidemiological studies: a review. J. R. Soc. Med., 85: 165-168.

20. KLINK E.G.M.V., CORTEN J.J.F.M., KALOKONI D.M., 1996. Herd monitoring in traditional cattle husbandry as a tool for productivity research and livestock development. Trop. Anim. Health Prod., 28: 273-279.

21. KSIAZEK T.G., JOUAN A., MEEGAN J.M., LE GUENNO B., WILSON M.L., PETERS C.J., DIGOUTTE J.P., GUILLAUD M., MERZOU G N.O., TOURAY E.M., 1989. Rift Valley fever among domestic animals in the recent W est African outbreak. Res. Virol., 140: 67-77.

22. LANDAIS E., LHOSTE P., MILLEVILLE P., 1987. Points de vue sur la zootechnie et les systèmes d'élevage tropicaux. Cah. Sci. hum., 3-4: 421-437.

23. LANDAIS E., SISSO KHO M.M., 1986. Bases méthodologiques du contrôle des performances animales pour l'analyse zootechnique et démographique : collecte des données et choix des variables. In : Landais $E$., Faye B. ed., Méthodes pour la recherche sur les systèmes d'élevage en Afrique intertropicale, Mbour (Sénégal). Maisons-Alfort, France, Cirad-lemvt, p. 433-485. (Etudes et synthèses)
24. LEEU W P.N. de, M CDERM OTT J.J., LEBBIE S.H.B., 1995. Monitoring of livestock health and production in sub-saharan Africa. Prev. vet. Med., 22: 195-212.

25. LEFEVRE-DESO UTTER D., 1994. Les pneumopathies des petits ruminants au Sénégal : étude séro-épidémiologique et bio-géographique. Thèse doct d'université, Université Paris XII, Créteil, France, 189 p. + annexes.

26. LESCOURRET F., DORR N., FAYE B., 1994. Conception et intérêts d'une base de données dans un système d'information à visée de recherche exploratoire : un exemple en épidémiologie animale. In : 2nd int. Symp. Livest. Farming Syst. The study of livestock farming systems in a research and development framework, Saragossa, Spain, September 11-12, 1992, p. 207-211

27. LESCOURRET F., GENEST M., BARNOUIN J., CHASSAGNE M. FAYE B., 1993. Data modelling for database design in production and health monitoring systems for dairy herds. J. Dairy Sci., 76: 1053-1062.

28. LESCOURRET F., PEROCHON L., COULON J.-B., FAYE B., LANDAIS E., 1992. Modelling an information system using the Merise method for agricultural research: the example of a database for a study on performances in dairy cows. Agric. Sys., 38: 149-173.

29. LESN OFF M., LANCELOT R., 1997. Estimation and impact of the measurement errors for small ruminants demographic data collected during retrospective interview of farmers in Senegal. Épidémiol. Santé Anim., 31-32: 02.15.1-02.15.3.

30. LHOSTE P., 1986. L'association agriculture-élevage. Evolution du système agropastoral du Siné-Saloum (Sénégal). Thèse doct. Ingénieur Sci. agron., Ina, Paris - Grignon, France, 314 p.

31. LO M., 1991. Résultats du suivi zootechnique au Projet de développement de l'élevage ovin. Dakar, Sénégal, Lnerv, 98 p.

32. MARCHAL V., 1997. Analyses des performances pondérales des bovins allaitants en Province Nord de Nouvelle Calédonie (Mandat de gestion N ouvelle Calédonie). Pouembout, N ouvelle Calédonie, Cirad, $40 \mathrm{p}$.

33. MARCHAL V., 1997. Analyses des performances pondérales des bovins allaitants en Province Nord de Nouvelle Calédonie. Première partie : les poids à âges types (Mandat de gestion Nouvelle-Calédonie). Pouembout, N ouvelle Calédonie, Cirad, $48 \mathrm{p}$.

34. MATTHEWMAN R.W., 1980. Small ruminant production in the humid tropical zone of southern Nigeria. Trop. Anim. Health Prod., 12: 234-342.

35. MOULIN C.H., 1993. Performances animales et pratiques d'élevage en Afrique sahélienne. La diversité du fonctionnement des troupeaux de petits ruminants dans la Communauté rurale de $\mathrm{N}$ diagne (Sénégal). Thèse doct. Ingénieur Sci. agron., Ina, Paris - Grignon, France, $259 \mathrm{p}$.

36. MOU LIN C.H., FAU GERE O., FAU GERE B., 1994. L'élevage traditionnel des petits ruminants au Sénégal. III. Pratiques de conduite et d'exploitation des animaux chez les éleveurs de la communauté rurale de Kaymor (Siné-Saloum, Sénégal). Revue Elev. Méd. vét. Pays trop., $47: 223-234$

37. NDIAYE M., AKAKPO J.A., PANGUI L.J., 1994. Reproduction losses, chlamydiosis, toxoplasmosis and brucellosis in extensive traditional guinean flocks in Senegal. Kenya Vet., 18: 135

38. NGERE L.O., MBAP S., 1982. Aspects of reproduction in West African Dwarf goats. Trop. Anim. Health Prod., 14: 61.

39. OPPONG E.N.W., YEBUAH N.M.N., 1981. Some production traits of the West African D warf goat. Trop. Anim. Health Prod., 13: 208-212.

40. OSUAGWUH A.I.A., AKPOKODJE J.U., 1981. West African Dwarf (Foulta djallon) goat. 1. Causes of early mortality. Int. Goat Sheep Res. 1: 303-309.

41. PHARO H.J., SOPIAN M.J., KAMARUDDIN M., HASSAN M.A.A., CHEAH P.F., CHOO T.W., 1990. Progress in the use of computerised recording systems in dairy cow monitoring and extension in Malaysia. Trop. Anim. Health Prod., 22: 77-88.

42. POIVEY J.P., SEITZ J.L., LANDAIS E., 1981. Finalités et aspects méthodologiques d'un système informatisé de suivi individuel des animaux dans les élevages bovins villageois du Nord de la Côte d'Ivoire. Revue Elev. Méd. vét. Pays trop., 34 : 199-210.

43. ROMBAUT D., VLAENDEREN G. VAN, 1976. Le mouton Djallonké en Côte d'Ivoire en milieu villageois : comportement et alimentation. Revue Elev. Méd. vét. Pays trop., 29 : 157-172

44. ROWLANDS G.J., 1983. Data standards for computer systems in livestock recording for dairy herd management. Livest. Prod. Sci. 10: $419-429$. 
45. SALAMI I., 1990. Détermination de l'âge des petits ruminants par l'examen de la dentition. Elaboration et utilisation des grilles d'évaluation au Sénégal. Thèse doct. vét., EISM V, Université Cheikh Anta Diop, Dakar, Sénégal, $113 p$.

46. STONE D.J.W., THRUSFIELD M.V., 1989. A small animal clinical and epidemiological relational database. Prev. vet. Med., 7: 289-302.

47. SU LPICE P., LESCO URRET F., CALAVAS D., BU GNARD F., FAYE B., 1994. La gestion de l'information dans les enquêtes d'épidémiologie animale. Epidémiol. Santé Anim., 25 : 113-132.

48. TARDIEU H., ROCHFELD A., COLETTI R., 1983. La méthode Merise, tome I. Principes et outils. Paris, France, Edition d'organisation, $285 \mathrm{p}$.

49. TILLARD E., MOULIN C.-H., FAU GERE O., FAU GERE B., 1997. Le suivi individuel des petits ruminants au Sénégal : un mode d'étude des troupeaux en milieu villageois. Inra Prod. Anim., $10: 67-78$.

50. TOURRAND J.-F., 1993. L'élevage dans la révolution agricole au Waalo : ruptures et continuité. Thèse doct. d'état, U niversité Paris XII Créteil, France, $416 \mathrm{p}$.

51. TO URRAND J.-F., LANDAIS E., 1996. Productivité des caprins dans les systèmes de production agricole du Delta du fleuve Sénégal. Revue Elev. M éd. vét. Pays trop., 49 : 168-173.

52. UDOMPRASERT P., WILLIAMSON N.B., 1990. The DairyChamp program: a computerised recording system for dairy herds. Vet. Rec., (127): 256-262

\section{Summary}

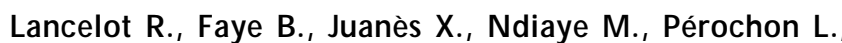
Tillard E. The Baobab database: a tool for modeling small ruminants production and health in traditional farming systems in Senegal

A relational database called Baobab was set up in order to facilitate data summarizing and modeling on small ruminants production and health in smallholding farms. It compiles data collected in four sites during a farm monitoring period of 15 years using Panurge information system. The sites were representative of the main agroecological areas in sheep and goat husbandry. Specific animal data were related to demography, weight increase and health. Other data were recorded at the herd level and concerned farming practices and disease control collective measures. The conceptual data model (cdm) was designed according to the Merise entityrelationship method. The database structure derived from the cdm. Panurge data were imported into Baobab using checking computer programs. Baobab covers 79,000 surveyed animals, includes 28 tables and takes 50 megabytes. A new application was set up from cdm. It will facilitate entering and use of data issued from individual animal monitoring in settled or transhumant herds. Baobab many applications can be presented along two main lines: 1) reference and summed-up data production that are useful to farmers' organizations, development projects and help compare countries or agroecological areas among themselves; 2) animal production modeling at the individual level (genetics, epidemiology) or the herd level (population dynamics, decision-making tool).

Key words: Sheep - Goat - Database - Information system Model - Productivity - Animal health - Senegal.
53. VIN CENT J.P., AN GIBAULT J.M., BIDEAU E., GAILLARD J.M., 1994. Le problème de la détermination de l'âge : une source d'erreur négligée dans le calcul des tables de vie transversales. Mammalia, 58 : 293-299.

54. WILSON R.T., 1976. Studies on the livestock of southern Darfur, Sudan. III. Production traits in sheep. Trop. Anim. Health Prod., 8: 103-114.

55. WILSO N R.T., 1976. Studies on the livestock of southern Darfur, Sudan. IV. Production traits in goats. Trop. Anim. Health Prod., 8: 221-232.

56. WILSO N R.T., 1980. Population and production parameters of sheep under traditional management in semi-arid areas of Africa. Trop. Anim. Health Prod., 12: 243-250.

57. WILSON R.T., 1983. Livestock production in Central Mali. The Macina wool sheep of the Niger inundation zone. Trop. Anim. Health Prod., 15: 17-31.

58. WILSON R.T., 1983. Studies on the livestock of southern Darfur, Sudan. VIII. A comparison of productivity indices for goats and sheep. Trop. Anim. Health Prod., 15: 63-68.

59. WILSO N R.T., DURKIN J.W., 1983. Livestock production in central Mali: weight at first conception and ages at first and second parturitions in traditionally managed goats and sheep. J. Agric. Sci. Camb., 100: 625-628.

Reçu le 15.4.98, accepté le 17.8.98

\section{Resumen}

Lancelot R., Faye B., Juanès $X_{\text {., }} N$ diaye $M$. ., Pérochon L., Tillard E. Base de datos Baobab: un instrumento para realizar modelos de la producción y de salud de los pequeños rumiantes en los sistemas de crianza tradicionales de Senegal

La base de datos relacionales Baobab se construyó para facilitar el trabajo de síntesis y de modelización sobre la producción y la salud de los pequeños rumiantes en crianza sedentaria tradicional en Senegal. Reune los datos recolectados en cuatro sitios representativos de las principales zonas agro ecológicas de crianza ovina y caprina, durante 15 años de seguimiento de los establecimientos, utilizando el sistema de información Panurge. Los datos individuales son relativos a la demografía, al crecimiento ponderal y a la salud. Los datos sobre las prácticas de manejo y las profilaxis colectivas fueron recolectados a nivel del hato. El modelo conceptual de datos (Mcd) se elaboró según el método entidad-relación Merise. La estructura de la base de datos se dedujo del Mcd. Los datos fueron importados luego a Panurge, en la nueva base de datos, gracias a programas con procedimientos correctivos y de control. Baobab posee 28 tablas y ocupa un espacio de disco de alrededor 50 Mo para 79000 animales censados. Se derivó una nueva aplicación del Mcd. Esta facilitará la extracción y el análisis de los datos provenientes de seguimientos zootécnicos individuales, en sistemas sedentarios o trashumantes. Las perspectivas de utilización de Baobab son numerosas y se orientan según dos ejes principales: 1) la producción de referencias y síntesis utilizables por las organizaciones de productores y los proyectos de desarrollo, permitiendo la comparación entre el país y las zonas agro ecológicas; 2) la modelización de la producción animal a nivel individual (genética, epidemiológica) o del hato (dinámica de la población, ayuda en la toma de decisiones).

Palabras clave: O vino - Caprino - Base de datos - Sistema de información - Modelo - Productividad - Sanidad animal Senegal. 